\title{
Predictive Analysis in Decompressive Craniotomy for Haemorrhagic Stroke
}

\author{
Md Moshiur Rahman ${ }^{1 *}$, K M Ziaur Rahman² and Imrul Kaies ${ }^{3}$ \\ ${ }^{1}$ Assistant Professor, Neurosurgery Department, Holy Family Red Crescent Medical \\ College, Dhaka, Bangladesh \\ ${ }^{2}$ Resident, Neurosurgery Department, Holy Family Red Crescent Medical College, \\ Dhaka, Bangladesh \\ ${ }^{3}$ Medical Officer, Anaesthesia Department, Bangabandhu Sheikh Mujib Medical \\ University (BSMMU), Dhaka \\ *Corresponding Author: Md Moshiur Rahman, Assistant Professor, Neurosurgery \\ Department, Holy Family Red Crescent Medical College, Dhaka, Bangladesh.
}

\begin{abstract}
Background: Haematoma volume is a strong predictor of morbidity and mortality in a spontaneous intracerebral hematoma. Timing of surgery, amount of clot removal, GCS on admission, pupillary abnormality and amount of bone removal of such cases are strong variables. A large amount of blood is causing impending herniation which is life-threatening and should be addressed immediately to reverse the situation.

Objective: The main goal of this study is to assess the predictive analysis in decompressive craniotomy for haemorrhagic stroke.

Method: A total of 72 cases were included in this study. This retrospective study was conducted in three private hospitals from 2009 to 2018. Male: Female was 3:2. Surgical outcome predictors were analyzed by using different variables- the timing of surgery, amount of clot removal, GCS on admission, pupillary abnormality, age of the patients and amount of bone removal.

Results: 8 patients died, 2 patients were in a vegetative state, 1 patient developed osteomyelitis in a bone flap and 1 had CSF leak and meningitis.

Conclusion: Decompressive craniotomy for large intracerebral hematoma is lifesaving. Among the variables- the timing of surgery and the amount of bone removal are strong predictors of the outcome of the surgery.
\end{abstract}

Keywords: Haematoma; Craniotomy; Decompressive; Intracerebral

\section{Abbreviations}

SAH: Subarachnoid Haemorrhage; ICH: Intracerebral Haemorrhage; CP: Cranioplasty; DC: Decompressive Craniectomy; CSF: Cerebrospinal Fluid; ICP: Intracranial Pressure; GCS: Glasgow Coma Scale; GOS: Glasgow Outcome Scale

\section{Introduction}

One can differentiate between two clinical settings that could be known as DC. For patients with elevated intracranial pressure due to brain swelling due to traumatic brain injury, cerebral infarction, subarachnoid haemorrhage (SAH), intracerebral hemorrhage (ICH) and for other purposes, decompressive craniectomy (DC) is often performed as a potentially life-saving procedure. The wi- der range of indications for DC in survivors suggests an increasing number of subsequent cranioplasty (CP) procedures. In addition, $\mathrm{CP}$ has been documented to promote neurological recovery and enhance cerebral blood flow, hydrodynamics of the cerebrospinal fluid (CSF), and metabolic activity following a decompressive craniectomy [1]. The main purpose of the neurosurgical procedure in this setting is bone removal. DC is a matter of debate, after such a process. The second clinical situation is the patient who has a late clinical decline associated with brain swelling. The DC may have some interesting cases of medical treatment failed to prevent resulting brain death. In life-threatening intracranial pathologies such as intracerebral bleeding and traumatic or ischemic brain swelling, a decompressive craniotomy may be seen as a therapeutic alterna- 
tive when intensive medical management is not adequate to relieve the intracranial pressure (ICP) [2-4]. Although the procedure, its complications and the potential benefit of decompressive cranial surgery have been examined extensively, there are still many open questions about the subsequent remodeling of the skull. In addition, it is important to elucidate the occurrence and function of potential long-term complications, which require repeated surgical procedures.

\section{Objective}

Our goal of this study is to assess the predictive analysis in decompressive craniotomy for haemorrhagic stroke.

\section{Methodology}

Study type

- $\quad$ This study was a retrospective study.

Study place

- $\quad$ This study was conducted in three private hospitals.

Study period

- $\quad$ From 2009 to 2018.

Sample size

- $\quad$ Total of 72 cases.

Inclusion criteria

- $\quad$ Patients having GCS 4 or more.

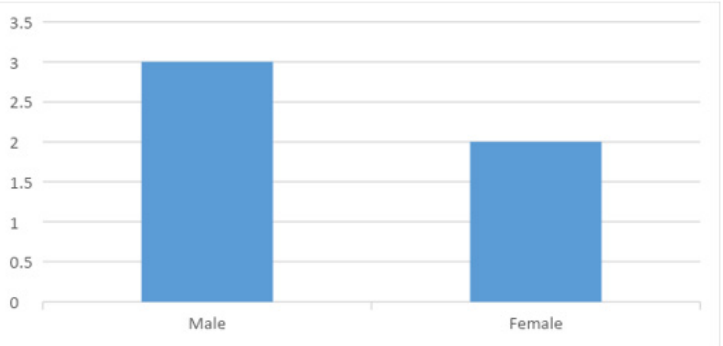

Figure 1: The ratio of male and female (3:2).

\section{Study procedure}

Surgical outcome predictors were analyzed by using different variables- age of the patients, the timing of surgery, amount of clot removal, GCS on admission, pupillary abnormality, ICH score, GOS and amount of bone removal.

\section{Results}

Characteristics of the patients

The total number of patients was 72 in our study. Among them 54 were male and 18 were female patients. The maximum age of the patients was 80 .

Table 1 and 2 is showing that maximum patients had normal pupil but 14 patients had extended right pupil and 19 patients had extended left pupil with poor reaction to light.

\begin{tabular}{|l|c|c|}
\hline Age in years & Male & Female \\
\hline$<30$ & 3 & 1 \\
\hline $30-40$ & 8 & 2 \\
\hline $40-50$ & 16 & 9 \\
\hline $50-60$ & 11 & 3 \\
\hline $60-70$ & 9 & 2 \\
\hline $70-80$ & 7 & 1 \\
\hline Total & 54 & 18 \\
\hline
\end{tabular}

Table 1: Age and gender distribution $(n=72)$.

\begin{tabular}{|l|c|}
\hline Pupil abnormality & Patient number \\
\hline $\begin{array}{l}\text { Right pupil extended and poor reaction to } \\
\text { light, OCR-normal }\end{array}$ & 14 \\
\hline $\begin{array}{l}\text { Left pupil extended and poor reaction to } \\
\text { light, OCR-normal }\end{array}$ & 19 \\
\hline Normal pupil reaction, OCR-normal & 39 \\
\hline
\end{tabular}

Table 2: Pupil abnormality $(n=72)$.

Figure 2 shows that 29 patients had surgery within four hours of admission and 43 patients had surgery after four hours.

After 4 hours 
In our study we included patients with GCS 4 or more on admission and maximum of them gad GCS 4-6 on scale. And table 3 shows that in our study the maximum number of patients had a good outcome of surgery according to the Glasgow outcome scale.

\begin{tabular}{|l|c|}
\hline Outcome & Number of patients \\
\hline Good & 40 \\
\hline Adequate disability & 12 \\
\hline Acute disability & 10 \\
\hline Vegetative & 2 \\
\hline Dead & 8 \\
\hline
\end{tabular}

Table 3: Outcome of surgery, GOS (Glasgow Outcome Scale) $(\mathrm{n}=72)$.

Table 4 is showing the amount of clot that we were removed from patients.

\begin{tabular}{|c|c|}
\hline Number of patients & Amount of clot removal ((cm $\mathbf{3})$ \\
\hline 7 & $45-60$ \\
\hline 12 & $60-75$ \\
\hline 9 & $75-90$ \\
\hline
\end{tabular}

Table 4: Amount of clot removal.

Table 5 shows the ICH score of the patients who have undergone decompressive craniotomy. And maximum patients had ICH score around 2 - 3.

\begin{tabular}{|c|c|c|}
\hline ICH Score & Mortality risk & Number of patients \\
\hline 0 & $0 \%$ & 9 \\
\hline 1 & $13 \%$ & 11 \\
\hline 2 & $26 \%$ & 12 \\
\hline 3 & $72 \%$ & 27 \\
\hline 4 & $97 \%$ & 5 \\
\hline 5 & $100 \%$ & 8 \\
\hline 6 & $100 \%$ & 0 \\
\hline
\end{tabular}

Table 5: ICH score.

The size of bone flap removal in maximum patients was equal to or less than $60 \mathrm{~cm}^{2}$. See table 6 below.

\begin{tabular}{|l|c|}
\hline Bone size $\left(\mathbf{c m}^{2}\right)$ & Patients number \\
\hline$\leq 60$ & 28 \\
\hline $61-70$ & 14 \\
\hline $71-80$ & 17 \\
\hline $81-90$ & 13 \\
\hline
\end{tabular}

Table 6: Amount of bone removal.

\section{Complications}

2 patients were in a vegetative state, 1 patient developed osteomyelitis in a bone flap and 1 had CSF leak and meningitis. See figure 3 and 4 .

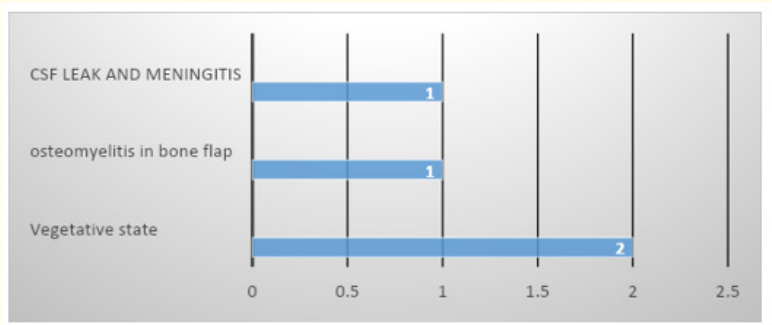

Figure 3: Complications level.

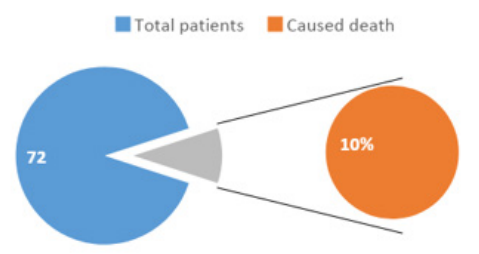

Figure 4: Mortality rate.

\section{Mortality rate}

Among 72 patients 8 patients were dead.

\section{Discussion}

In the present study, 72 patients admitted to the hospital were given decompressive craniotomy for large intracerebral haematoma. The findings of the current study indicate that $85 \%$ of the good recovery in patients was correlated with DC. Eight patients died in our study, and 4 patients suffered minor complications. Of these 4, 2 remained in a vegetative state, 1 had CSF leakage and meningitis, and 1 had bone flap osteomyelitis. According to these findings, early DC in serious haemorrhagic stroke patients should be recommended in patients needing neurosurgery to eliminate intracranial collection, where the absence of brain stem dysfunction is shown by the first clinical review. These data are in line with those obtained in a case-control study which demonstrated a strict and statistically significant correlation between initial GCS and performance [5]. Our study's results in GOS were good in 40 patients. ICH score consists of five ICH outcome-related components and is a validated ICH clinical rating scale that reliably risk-stratifies pati- 
ents with respect to 30 -day mortality. ICH score range is 0 to 6 , and each increase in the ICH score is correlated with a 30-day mortality rise [6,7]. All patients with an ICH score of $(0-4)$ have survived in our study and all patients with an ICH score of 5 died. 33 patients had extreme intracranial hypertension with a pupillary abnormality, immune to well-conducted medical treatment consisting of a type of cerebrospinal fluid. Such patients did personally undergo surgery during cerebral herniation. For maximum cases, a mild or no impairment was found at a 1-year follow-up, given their severity. The mean length of follow-up was 13.32 months (range: 1 - 24 months). Of the 33 patients with pupillary defects, 27 recovered well. Yet they did have natural occult-cephalic reflexes, which produced good results. Earlier studies have shown that following treatment, an irregular occult-cephalic reflex is associated with poor prognosis when present in patients with hemispheric ICH. Effective recovery is possible, even in the presence of nonreactive pupils at mid-position [8]. In our series the risk of meningitis or cerebral abscess is not as high. One theory is that patients with a DC obtained antibiotics for sepsis syndrome, as a result of which the diagnosis of meningitis was understated. On the other hand, our meningitis rate could have been overestimated, as we have taken into account all cases of inflammation of the cerebrospinal fluid, including potentially chemical meningitis [9]. In an ICP-related infection sample, 68 test tips were cultivated: $13.2 \%$ had a positive culture without clinical signs of infection and $2.9 \%$ had a positive culture with clinical signs of ventriculitis [10]. Staphylococcus epidermidis was the most prevalent isolated pathogen. In this sense, it may be surprising that our patients studied after DC and in most studies lack infection. DC is a rescue technique that should be considered at the time of hospital admission in the event of medical treatment failure, especially in young patients without brain stem dysfunctions. Because of brain edema, this procedure may prevent cerebral herniation associated with intracranial hypertension. There are certain drawbacks to our research that need to be acknowledged. This series of decompressive craniectomy by evacuation of hematoma is small and is performed in a select group of patients. It is possible that surgery, as indicated by the predominance of right hemispheric $\mathrm{ICH}$, was given only to those patients who claimed to have a greater chance of recovery. Given these limitations, our study suggests that hematoma-evacuated decompressive craniectomy is life-saving, and its functional recovery may be good. But findings may not be good when there is clinical evidence of compression of the brainstem. Because this is a retrospective small series of data analyses, further studies have to equate patients with surgery in a prospective, randomized manner to conservative treatment.

\section{Conclusion}

DC is a major treatment option in all age groups for stroke. A significant reduction in mortality was reported by DC for haemorrhagic stroke. Nonetheless, DC makes a significant proportion of patients with a moderately severe disability. Accurate awareness of the relevant data is thus important for individual patients in the decision-making process. An even greater challenge could be to determine whether the patient, based on preoperative expectations, will have a reasonable impairment and quality of life in his or her assumed understanding. Future studies will strive to improve our expertise and evidence base on DC and should adjust to the personalized medicine model by predicting more reliably when and how to perform DC in different patients to achieve optimum results.

\section{Acknowledgements}

The biggest acknowledgement goes to the patients who have taken participate in this study and also to everyone who makes a contribution to make this research possible.

\section{Patient Consent}

The patient provided written informed consent for the publication and the use of their images.

Financial Support/Sponsorship

Nil.

Conflicts of Interest

Nil.

\section{Bibliography}

1. Honeybul S., et al. "The impact of cranioplasty on neurological function". British Journal of Neurosurgery 27 (2013): 636-641.

2. Meyer MJ., et al. "Acute management of acquired brain injury part I: an evidence-based review of non-pharmacological interventions". Brain Injury 24 (2010): 694-705.

3. Sahuquillo J., et al. "Decompressive craniectomy for the treatment of refractory high intracranial pressure in traumatic brain injury". Cochrane Database of Systematic Reviews 1 (2006): CD003983.

4. Schwab S., et al. "Early hemicraniectomy in patients with complete middle cer ebral artery infarction”. Stroke 29 (1998): 1888-1893. 
5. Polin RS., et al. "Decompressive bifrontal craniectomy in the treatment of severe refractory posttraumatic cerebral edema". Neurosurgery 41 (1997): 84-92.

6. Hamphill JC., et al. "The ICH score: a simple, reliable grading scale for intracerebral haemorrhage". Stroke 32 (2001): 891897.

7. Kothari RU., et al. "The ABCs of measuring intracerebral hemorrhage volumes”. Stroke 27 (1996): 1304-1305.

8. Rabinstein AA., et al. "Emergency craniotomy in patients worsening due to expanded cerebral hematoma: to what purpose?" Neurology 58 (2002): 1367-1372.

9. Forgacs P., et al. "Characterization of chemical meningitis after neurological surgery”. Clinical Infectious Diseases 32 (2001): 179-185.

10. Martinez-Manas RM., et al. "Camino intracranial pressure monitor: Prospective study of accuracy and complications". Journal of Neurology, Neurosurgery, and Psychiatry 69 (2000): 82-86.

\section{Assets from publication with us}

- Prompt Acknowledgement after receiving the article

- Thorough Double blinded peer review

- Rapid Publication

- Issue of Publication Certificate

- High visibility of your Published work

Website: https://www.actascientific.com/

Submit Article: https://www.actascientific.com/submission.php Email us: editor@actascientific.com

Contact us: +919182824667 